\title{
A case of early stage lung cancer detected by repeated cancer screening with positron emission tomography
}

\author{
IKUKO SAKATA ${ }^{1}$, YUICHI OZEKI ${ }^{2}$, KATSUMI TAMURA ${ }^{1}$, \\ JIRO ISHIDA $^{1}$, SHINSUKE AIDA ${ }^{3}$ and YOSHIYUKI ABE ${ }^{1}$ \\ ${ }^{1}$ Tokorozawa PET Diagnostic Imaging Clinic; \\ Departments of ${ }^{2}$ Thoracic Surgery and ${ }^{3}$ Diagnostic Pathology, \\ National Defense Medical College, Tokorozawa, Saitama 359-1124, Japan
}

Received September 23, 2011; Accepted November 7, 2011

DOI: $10.3892 / \mathrm{ol} .2011 .492$

\begin{abstract}
There has been an increase in the detection rate of small early lung cancer due to recent improvements in imaging technology. However, conventional imaging modalities such as computed tomography (CT) alone are not capable of differentiating small pulmonary nodules. New modalities such as F-18 2'-deoxy-2fluoro-D-glucose (FDG) positron emission tomography combined with $\mathrm{CT}$ (PET/CT) have contributed to the evaluation of lung cancer staging, although the differential diagnosis of pulmonary nodules showing ground-glass opacity (GGO) with PET/CT is controversial. In Japan, cancer screening with whole body FDG-PET has been available for asymptomatic individuals, and it has been reported that a wide variety of cancer types are detectable by FDG-PET at potentially curable stages. We present the case of a 62-year-old male with early lung cancer, which was revealed by repeated health screening. A PET/CT scan revealed definite intense FDG uptake (SUVmax 1.2) in the pulmonary nodules of the right upper lobe, while no definite FDG uptake was observed in the lesion in the previous annual screening. Right upper lobectomy was performed, and the pathological diagnosis was well-differentiated adenocarcinoma. Five-year survival has been noted since the thoracotomy, and the patient is doing well without recurrence. This is a significant case of early lung cancer with GGO lesions, which revealed intense FDG uptake during an annual repeated health screening with FDG-PET/CT.
\end{abstract}

\section{Introduction}

An increase has been noted in the rate of detection of small early lung cancers with recent improvements in imaging tech-

Correspondence to: Dr Yoshiyuki Abe, Tokorozawa PET Diagnostic Imaging Clinic, Higashisumiyoshi 7-5, Tokorozawa, Saitama 359-1124, Japan

E-mail: abe@toko-pet.or.jp

Key words: lung cancer, positron emission tomography/computed tomography, screening, ground-glass opacity nology such as computed tomography (CT). In particular, since high-resolution computed tomography (HRCT) scans with low radiation dose were first applied for lung cancer screening during the late 1990s to 2000s (1-3), there has been a marked increase in the detection of ground-glass opacity (GGO) in peripheral lung lesions. GGO is a non-specific finding that may be caused by various diseases, including inflammation, fibrosis and cancer, while other studies have reported that GGO is related to bronchioalveolar carcinoma (BAC) (4).

Positron emission tomography (PET) with F-18 2'-deoxy2fluoro-D-glucose (FDG) has been used to differentiate malignant from benign lesions due to the higher metabolic activity of malignant lesions indicated by high secondary isotope uptake. Numerous reports are available regarding the usefulness of FDG-PET in differentiating malignant pulmonary nodules from benign ones $(5,6)$. In Japan, cancer screening with whole FDG-PET has been available for asymptomatic individuals, albeit with a high procedure fee. Moreover, it has been reported that a wide variety of cancer types are detectable by FDG-PET at potentially curable stages (7-9). New modalities such as FDG-PET combined with computed tomography (FDG-PET/CT) are now well-established for the evaluation of various cancer types (10-13). Clinically, PET/CT has contributed to the evaluation of lung cancer staging, while the usefulness of FDG-PET/CT for the differential diagnosis in small nodules showing GGO remains controversial (14-17).

We report a noteworthy case of early lung cancer with GGO lesions, which revealed definite intense FDG uptake during repeated health screening with a PET/CT scan.

\section{Patient and methods}

This study was performed with the patient's informed consent and with approval for the study from the ethics committee of Tokorozawa PET Diagnostic Imaging Clinic, Japan.

A 62-year-old man received health screening, including a PET/CT scan, in September 2005 at Tokorozawa PET Diagnostic Imaging Clinic. ${ }^{18} \mathrm{~F}-\mathrm{FDG}$ PET/CT scans were obtained with a Biograph Duo (Siemens CTI) as described in our previous study $(11,18)$. To determine semi-quantitative FDG uptake, regions of interest (ROIs) were placed over the lesion, including the highest uptake area (circular ROI, $1 \mathrm{~cm}$ 
A

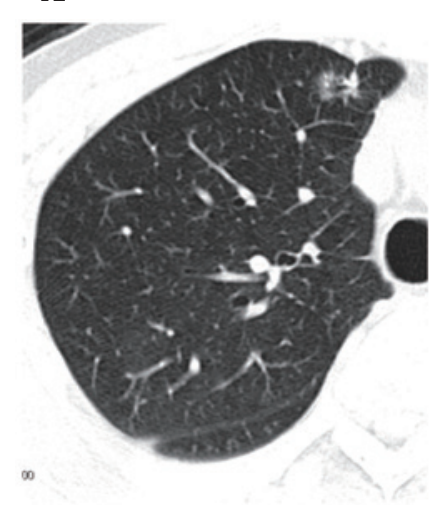

C

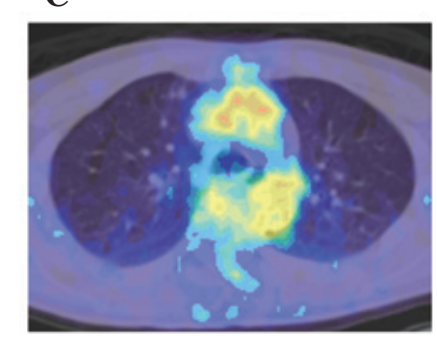

B

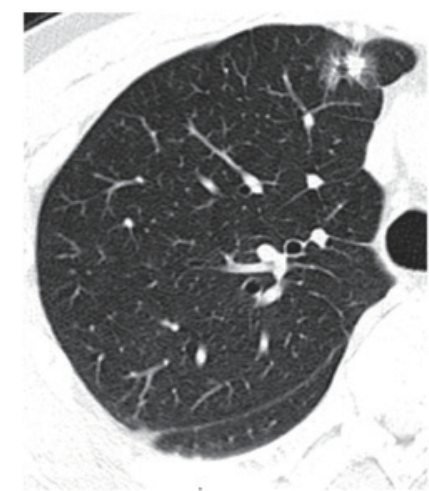

D

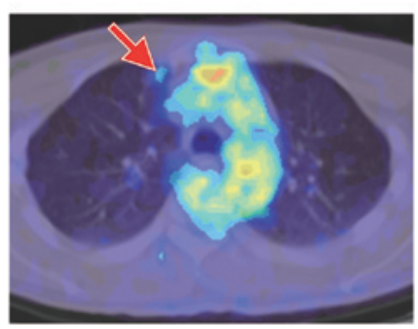

Figure 1. (A) A CT scan revealed a ground-glass opacity (GGO) lesion $(10 \mathrm{~mm})$ in the upper lobe of the right lung of a patient in September 2005. (B) A year later, the CT scan revealed an abnormal shadow of a larger size $(15 \mathrm{~mm})$ in the upper lobe of the right lung and GGO with a small solid area (C) PET/CT revealed no abnormal F-18 2'-deoxy-2fluoro-D-glucose (FDG) uptake in the GGO lesion in September 2005. (D) PET/CT revealed abnormal FDG uptake in the GGO lesion with SUVmax 1.2 (arrow) one year later.

in diameter), and the standardized uptake value (SUV) was calculated. The CT scan revealed a GGO lesion $(10 \mathrm{~mm})$ in the upper lobe of the right lung (Fig. 1A). PET/CT revealed no abnormal FDG uptake in this GGO lesion (Fig. 1C), and also suggested no apparent malignant findings in the whole body. Physical examination revealed no apparent abnormal findings, and no abnormalities were revealed in the blood analysis, including tumor markers such as carcinoembryonic antigen (CEA) and CA19-9. The patient consulted a chest surgeon but no explanatory thoracotomy was performed at the patient's request. Three months later, the patient received another CT scan but no change was noted in the abnormal shadow of the right upper lobe. A year later, he again underwent health screening with a PET/CT scan. The CT scan revealed a larger size $(15 \mathrm{~mm})$ abnormal shadow in the upper lobe of the right lung, and a small solid area with pleural indentation was noted in the GGO lesion (Fig. 1B). PET/CT revealed abnormal FDG uptake in this GGO lesion with SUVmax 1.2 (Fig. 1D).

\section{Results}

Explanatory thoracotomy was performed and biopsy specimens were obtained at the National Defense Medical College Hospital, Japan. Frozen sections of the tumor revealed a growth of cancer cells. The patient underwent right upper lobectomy plus dissection of the hilar and mediastinal lymph nodes. Macroscopically, the tumor in the right upper lobe measured $13 \times 13 \times 10 \mathrm{~mm}$ in diameter, and the cut surface of the tumor was

A

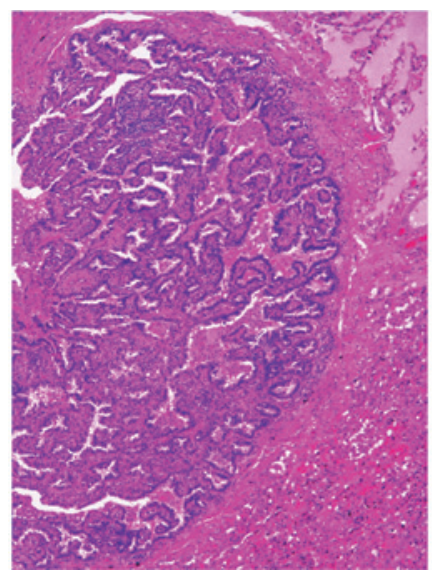

B

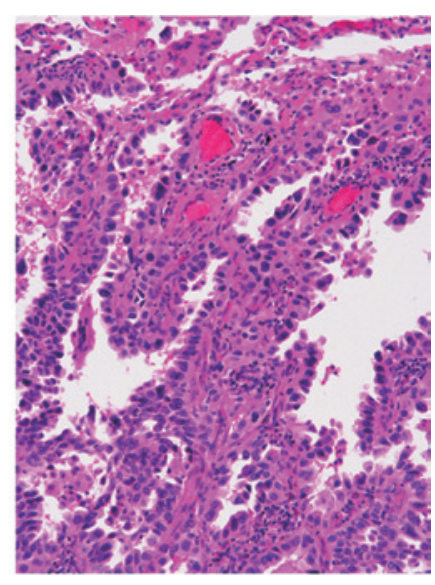

Figure 2. (A) Microscopic examination of the right pulmonary tumor lesion showed foci of tumor growth of atypical cells (magnification, x100). (B) Microscopic findings of the right pulmonary tumor on high power view revealed tumor cells with a glandular and papillary pattern with fibroblastic formation, a feature of well-differentiated adenocarcinoma; Noguchi classification type $\mathrm{C}$ (magnification, $\mathrm{x} 500$ ).

solid with a blue-white color. The microscopic findings revealed growth of atypical cells with a glandular and papillary pattern with fibroblastic formation, a feature of well-differentiated adenocarcinoma (Noguchi classification type C; pT1NOMO; Fig. 2). No metastatic lesions were observed in the dissected lymph nodes and the patient received no chemotherapy following the surgery. Five years after thoracotomy, PET/CT revealed no abnormalities and there were no signs of either recurrence or systemic metastasis in any other examinations.

\section{Discussion}

Low-dose HRCT scans were first applied for lung cancer screening between the late 1990s and early 2000s (1-3). In their study, Henschke et al reported that CT screening significantly reduced lung cancer mortality in a cohort of approximately 8,000 smokers (19). The development of FDG-PET/CT has contributed to the evaluation of human cancer staging, and the usefulness of PET/CT is well established for cancer staging. This imaging modality increases the otolaryngologist's and radiation oncologist's confidence when treating head and neck cancer patients, leading to appropriate management changes (20). We have reported that this modality was clinically useful for evaluating human cancers, including rare carcinoma cases (18,21-23). In the present study, we reported a lung cancer case with GGO, which revealed an increased intense FDG uptake by FDG-PET/CT during annual cancer screening, and the repeated FDG-PET/CT examinations were used to evaluate the pulmonary nodules with GGO.

In lung cancer screening with low-dose HRCT, there has been a marked increase in the detection of GGO in peripheral lung lesions. Computer-aided diagnosis (CAD) systems provide a useful second opinion in detecting pulmonary nodules when physicians carry out lung cancer screening with low-dose HRCT (24). Certain reports indicate that CT findings on GGO with a solid area are useful in differentiating between benign and malignant lesions (25). In our case, the CT scan revealed that 
the pulmonary lesion with GGO had a slightly increased size with a solid area after a one year interval between screenings.

In Japan, cancer screening with whole FDG-PET has been available for use in asymptomatic individuals, albeit with high procedure fees, and it has been reported that a wide variety of cancer types are detectable by FDG-PET at potentially curable stages (7-9). Murano et al reported that FDG-PET cancer screening was beneficial to patients above the break-even age despite the exposure to radiation (26). In our PET center, we performed cancer screening by PET/ $\mathrm{CT}$ with informed consent including that for radiation exposure, and 140 cases of cancer were detected by PET/CT and other modalities among 7,236 examinations in a period of approximately 5 years and 7 months between August 2005 and March 2011. A total of 140 cancer patients were pathologically diagnosed as having various cancers: 33 lung, 17 thyroid, 17 breast, 11 colon and 62 other cancer types, whereas PET/CT revealed no significant FDG uptake in 22 of the 140 patients. The total detection rate was $1.93 \%$, and the detection rate with PET/CT was $1.55 \%$.

In this study, we have reported a rare case of early lung cancer with GGO lesions. The patient underwent thoracotomy and at present is without recurrence. Thus, intense FDG uptake during an annual follow-up repeated health screening with FDG-PET/CT may prove useful in detecting such cancer types.

\section{Acknowledgements}

The authors are grateful to Mr. Kenji Kawai for his technical assistance.

\section{References}

1. Kaneko M, Eguchi K, Ohmatsu H, Kakinuma R, Naruke T, Suemasu K and Moriyama N: Peripheral lung cancer: screening and detection with low-dose spiral CT versus radiography. Radiology 201: 798-802, 1996

2. Abe Y, Hanai K, Nakano M, Ohkubo Y, Hashizume T, Kakizaki T, Nakamura M, Niki N, Egichi K, Fujino T and Moriyama N: A computer-aided diagnosis (CAD) system in lung cancer screening with computed tomography. Anticancer Res 25: 483-488, 2005.

3. Abe Y, Nakamura M, Ozeki Y, Machida K and Ogata T: Lung cancer: Low-dose helical computed tomography. In: Cance Imaging: Lung and Breast Carcinoma. Vol 1. Hayat MA (ed). Elsevier Academic Press, pp203-207, 2007.

4. Nakata M, Saeki H, Takata I, Segawa Y, Mogami H, Mandai K and Eguchi K: Focal ground-glass opacity detected by low-dose helical CT. Chest 121: 1464-1467, 2002.

5. Gould MK, Maclean CC, Kuschner WG, Rydzak CE and Owens DK: Accuracy of positron emission tomography for diagnosis of pulmonary nodules and mass lesions: a metaanalysis. JAMA 285: 914-924, 2001.

6. Kim SK, Allen-Auerbach M, Goldin J, Fueger BJ, Dahlbom M, Brown M, Czernin J and Schiepers C: Accuracy of PET/CT in characterization of solitary pulmonary lesions. J Nucl Med 48: 214-220, 2007.

7. Yasuda S and Shohtsu A: Cancer screening with whole-body $18 \mathrm{~F}$-fluorodeoxyglucose positron-emission tomography. Lancet 350: 1819, 1997.

8. Yasuda S, Ide M, Fujii H, Nakahara T, Mochizuki Y, Takahashi W and Shohtsu A: Application of positron emission tomography imaging to cancer screening. Br J Cancer 83: 16071611,2000

9. Ide M: Cancer screening with FDG-PET. Q J Nucl Med Mol Imaging 50: 23-27, 2006.

10. Cronin CG, Swords R, Truong MT, Viswanathan C, Rohren E, Giles FJ, O'Dwyer M and Bruzzi JF: Clinical utility of PET/CT in lymphoma. AJR Am J Roentgenol 194: W91-W103, 2010.
11. Ueda S, Saeki T, Tsuda H, Shigekawa T, Omata J, Moriya T, Yamamoto J, Osaki A, Fujiuchi N, M Misumi, et al: 18F-fluorodeoxyglucose positron emission tomography optimizes neoadjuvant chemotherapy for primary breast cancer to achieve pathological complete response. Int J Clin Oncol: 2011 (E-pub ahead of print).

12. Xie L, Saynak M, Veeramachaneni NK, Fried DV, Jagtap MR, Chiu WK, Higginson DS, Lawrence MV, Khandani AH, Qaqish BF, Chen RC and Marks LB: Non-small cell lung cancer: Prognostic importance of positive FDG PET findings in the mediastinum for patients with $\mathrm{N} 0-\mathrm{N} 1$ disease at pathologic analysis. Radiology: 2011 (E-pub ahead of print).

13. Abe Y, Tamura K, Sakata, I, Ishida J, Ozeki Y, Tamura A, Uematsu K, Sakai H, Goya T, Kanazawa M and Machida K: Clinical implications of ${ }^{18} \mathrm{~F}$-fluorodeoxyglucose positron emission tomography/computed tomography $\left({ }^{18} \mathrm{~F}-\mathrm{FDG}\right.$ PET/ $\mathrm{CT}$ ) at delayed phase for diagnosis and prognosis of malignant pleural mesothelioma. Oncol Rep: 2011 Oct 24. doi: 10.3892/ or.2011.1520 (Epub ahead of print).

14. Chun EJ, Lee HJ, Kang WJ, Kim KG, Goo JM, Park CM and Lee $\mathrm{CH}$ : Differentiation between malignancy and inflammation in pulmonary ground-glass nodules: the feasibility of integrated (18)F-FDG PET/CT. Lung Cancer 65: 180-186, 2009.

15. Fischer B, Lassen U, Mortensen J, Larsen S, Loft A, Bertelsen A, Ravn J, Clementsen P, Høgholm A, Larsen K, et al: Preoperative staging of lung cancer with combined PET-CT. N Engl J Med 361: 32-39, 2009.

16. Cloran FJ, Banks KP, Song WS, Kim and Bradley YC: Limitations of dual time point PET in the assessment of lung nodules with low FDG avidity. Lung Cancer 68: 66-71, 2010.

17. Fischer BM, Lassen U and Højgaard L: PET-CT in preoperative staging of lung cancer. N Engl J Med 364: 980-981, 2011.

18. Abe Y, Tamura K, Sakata I, Ishida J, Fukuba I, Matsuoka R, Shimizu S, Murakami H and Machida K: Usefulness of ${ }^{18} \mathrm{~F}-\mathrm{FDG}$ positron emission tomography/computed tomography for the diagnosis of pyothorax-associated lymphoma: A report of three cases. Oncol Lett 1: 833-836, 2010.

19. Henschke CI, Boffetta P, Gorlova O, Yip R, Delancey JO and Foy M: Assessment of lung-cancer mortality reduction from CT screening. Lung Cancer 71: 328-332, 2011.

20. Fleming AJ Jr and Johansen ME: The clinician's expectations from the use of positron emission tomography/computed tomography scanning in untreated and treated head and neck cancer patients. Curr Opin Otolaryngol Head Neck Surg 16: 127-134, 2008.

21. Ueda S, Tsuda H, Asakawa H, Shigekawa T, Fukatsu K, Kondo N, Yamamoto M, Hama Y, Tamura K, Ishida J, Abe Y and Mochizuki H: Clinicopathological and prognostic relevance of uptake level using 18F-fluorodeoxyglucose positron emission tomography computed tomography fusion imaging (18F-FDG PET/CT) in primary breast cancer. Jpn J Clin Oncol 38: 250-258, 2008.

22. Abe Y, Tamura K, Sakata, I, Ishida J, Mukai M, Ohtaki M, Nakamura $\mathrm{M}$ and Machida K: Unique intense uptake demonstrated by ${ }^{18} \mathrm{~F}$-FDG positron emission tomography/computed tomography (PET/CT) in primary pancreatic lymphoma: A case report. Oncol Lett 1: 605-607, 2010.

23. Ozeki Y, Abe Y, Kita H, Tamura K, Sakata, I, Ishida J and Machida K: A case of primary lung cancer lesion demonstrated by F-18 FDG positron emission tomography/computed tomography (PET/CT) one year after the detection of metastatic brain tumor. Oncol Lett 2: 621-623, 2011.

24. Abe Y, Tamura T, Sakata I, Ishida J, Nagata M, Machida K and Ogata T: Lung cancer: Computer-aided diagnosis with computed tomography. In: Cancer Imaging: Lung and Breast Carcinoma. Vol 1. Hayat MA (ed). Elsevier Academic Press, pp209-214, 2007.

25. Suzuki K, Kusumoto M, Watanabe S, Tsuchiya R and Asamura H: Radiologic classification of small adenocarcinoma of the lung: radiologic-pathologic correlation and its prognostic impact. Ann Thorac Surg 81: 413-419, 2006.

26. Murano T, Minamimoto R, Senda M, Uno K, Jinnouchi S, Fukuda H, Iinuma T, Tsukamoto E, Terauchi T, Yoshida T, et al: Radiation exposure and risk-benefit analysis in cancer screening using FDG-PET: results of a Japanese nationwide survey. Ann Nucl Med: 2011 (E-pub ahead of print). 